\title{
ON THE MAXIMAL ANGLE BETWEEN COPOSITIVE MATRICES
}

\author{
FELIX GOLDBERG* AND NAOMI SHAKED-MONDERER ${ }^{\dagger}$
}

\begin{abstract}
Hiriart-Urruty and Seeger have posed the problem of finding the maximal possible angle $\theta_{\max }\left(\mathcal{C}_{n}\right)$ between two copositive matrices of order $n$. They have proved that $\theta_{\max }\left(\mathcal{C}_{2}\right)=\frac{3}{4} \pi$ and conjectured that $\theta_{\max }\left(\mathcal{C}_{n}\right)$ is equal to $\frac{3}{4} \pi$ for all $n \geq 2$. In this note we disprove their conjecture by showing that $\lim _{n \rightarrow \infty} \theta_{\max }\left(\mathcal{C}_{n}\right)=\pi$. Our proof uses a construction from algebraic graph theory. We also consider the related problem of finding the maximal angle between a nonnegative matrix and a positive semidefinite matrix of the same order.
\end{abstract}

Key words. copositive matrix, convex cone, critical angle, strongly regular graph, symmetric nonnegative inverse eigenvalue problem

AMS subject classifications. 15A48,52A40,05E30.

1. Introduction. A matrix $A$ is called copositive if $x^{T} A x \geq 0$ for every vector $x \geq 0$. The set of $n \times n$ copositive matrices $\mathcal{C}_{n}$ is a closed convex cone in the space $\mathcal{S}_{n}$ of $n \times n$ symmetric matrices. By the definition, the cone $\mathcal{C}_{n}$ includes as subsets the cone $\mathcal{P}_{n}$ of positive semidefinite matrices and the cone $\mathcal{N}_{n}$ of symmetric nonnegative matrices of order $n$. Therefore, it is easy to see that $\mathcal{P}_{n}+\mathcal{N}_{n} \subseteq \mathcal{C}_{n}$.

In [7] Diananda proved that for $n \leq 4$ this set inclusion is in fact an equality, and also cited an example due to A. Horn that shows that for $n \geq 5$ there are copositive matrices which cannot be decomposed as a sum of a positive semidefinite and a nonnegative matrix (see also [12, p. 597]). In a remarkable recent paper [11] Hildebrand has described all extreme rays of $\mathcal{C}_{5}$, but very little is known about the structure of $\mathcal{C}_{n}$ for $n \geq 6$.

Understanding the structure of this cone is important, among other reasons, since many combinatorial and nonconvex quadratic optimization problems can be equivalently reformulated as linear problems over the cone $\mathcal{C}_{n}$ or its dual, the cone $\mathcal{C}_{n}^{*}$ of $n \times n$ completely positive matrices (i.e., matrices $A$ that possess a factorization $A=B B^{T}$, where $B \geq 0$ ). For more information about copositive matrices and copositive optimization we refer the reader to the recent surveys [12, 8, 4] and the references therein.

\footnotetext{
*Department of Mathematics, Technion-IIT, Technion City, Haifa 32000, Israel (felix.goldberg@gmail.com).

${ }^{\dagger}$ The Max Stern Yezreel Valley College, Yezreel Valley 19300, Israel (nomi@tx.technion.ac.il). This work was supported by grant no. G-18-304.2/2011 by the German-Israeli Foundation for Scientific Research and Development (GIF).
} 
This paper is dedicated to the solution of a problem posed by Hiriart-Urruty and Seeger in their survey [12:

What is the greatest possible angle between two matrices in $\mathcal{C}_{n}$ ?

The angle between vectors $u, v$ in an inner product space $V$ is:

$$
\angle(u, v)=\arccos \frac{\langle u, v\rangle}{\|u\| \cdot\|v\|} .
$$

Given a convex cone $K \subseteq V$, the maximal angle attained between two vectors in the cone $K$ is denoted $\theta_{\max }(K)$, and a pair of vectors attaining this angle is called antipodal. For the study of maximal angles of cones we refer to [13, 14].

Here we consider $V=\mathcal{S}_{n}$, with the standard inner product

$$
\langle A, B\rangle=\operatorname{Tr} A B
$$

and the norm associated with it, that is the Frobenius norm $\|A\|=\sqrt{\sum_{i, j=1}^{n}\left|a_{i j}\right|^{2}}$.

In [12] it was shown that $\theta_{\max }\left(\mathcal{C}_{2}\right)=\frac{3}{4} \pi$ and the unique pair of $2 \times 2$ matrices (up to multiplication by a positive scalar) that attains this angle was found. Furthermore, in [12, Remark 6.18] a somewhat hesitant conjecture was made to the effect that $\theta_{\max }\left(\mathcal{C}_{n}\right)=\frac{3}{4} \pi$ for all $n \geq 2$.

We show in this note that the authors of [12] were rightly apprehensive about the said conjecture, and that the correct asymptotic answer to their problem is:

$$
\lim _{n \rightarrow \infty} \theta_{\max }\left(\mathcal{C}_{n}\right)=\pi
$$

Note that the cone $\mathcal{C}_{n}$ is pointed, i.e., $\mathcal{C}_{n} \cap\left(-\mathcal{C}_{n}\right)=\{0\}$ [12, Proposition 1.2], and thus clearly $\theta_{\max }\left(\mathcal{C}_{n}\right)<\pi$ for every $n$.

For the proof, we consider the maximal angle between a positive semidefinite matrix and a nonnegative matrix of the same order $n$. Let us denote this maximal angle by $\gamma_{n}$, i.e.,

$$
\gamma_{n}=\max _{\substack{0 \neq X \in \mathcal{P}_{n} \\ 0 \neq Y \in \mathcal{N}_{n}}} \angle(X, Y)=\max _{\substack{X \in \mathcal{P}_{n}, Y \in \mathcal{N}_{n} \\\|X\|=\|Y\|=1}} \arccos \langle X, Y\rangle .
$$

This maximum exists, since both $\mathcal{N}_{n}$ and $\mathcal{P}_{n}$ are closed and their intersection with the unit sphere is compact. Then by the inclusion $\mathcal{P}_{n}+\mathcal{N}_{n} \subseteq \mathcal{C}_{n}$ we have

$$
\gamma_{n} \leq \theta_{\max }\left(\mathcal{P}_{n}+\mathcal{N}_{n}\right) \leq \theta_{\max }\left(\mathcal{C}_{n}\right)
$$

We prove our result on $\theta_{\max }\left(\mathcal{C}_{n}\right)$ by establishing 


\section{Theorem 1.}

$$
\lim _{n \rightarrow \infty} \gamma_{n}=\lim _{n \rightarrow \infty} \theta_{\max }\left(\mathcal{C}_{n}\right)=\pi
$$

This is achieved by constructing a sequence of pairs $\left(P_{k}, N_{k}\right), P_{k} \in \mathcal{P}_{n_{k}}$ and $N_{k} \in \mathcal{N}_{n_{k}}$, where the orders $n_{k}$ tend to infinity and such that $\angle\left(P_{k}, N_{k}\right) \rightarrow \pi$. Note that $\left\{\gamma_{n}\right\}$ is a non-decreasing sequence, since the angle between $N \in \mathcal{N}_{n}$ and $P \in \mathcal{P}_{n}$ is equal to the angle between $N \oplus 0 \in \mathcal{N}_{n+1}$ and $P \oplus 0 \in \mathcal{P}_{n+1}$.

As the problem of calculating or estimating $\gamma_{n}$ is interesting in its own right, we start in Section 2 with some initial results on this problem, finding $\gamma_{3}$ and $\gamma_{4}$. Though the geometry of the cones $\mathcal{P}_{n}$ and $\mathcal{N}_{n}$ is much better understood that that of $\mathcal{C}_{n}$, calculating $\gamma_{n}$ is a very difficult task for $n \geq 5$. We will offer an explanation for this phenomenon by showing that the determination of $\gamma_{n}$ is closely related to the symmetric nonnegative inverse eigenvalue problem (SNIEP). Details on SNIEP and related problems can be found in [2] and the references of [17.

The main result is stated and proved in Section 5 by a construction based on algebraic graph theory. The interceding Sections 3 , 4 are devoted to the introduction of the relevant tools from this theory, in order to keep this note self-contained, albeit tersely so. We conclude in Section 6 with some remarks.

2. The maximal angle between a positive semidefinite matrix and a nonnegative matrix. In this section we consider the problem of determining maximal angle between a positive semidefinite matrix and a nonnegative matrix of the same order for its own sake. However, the observations made in this section will also be instrumental in establishing the main result.

Every $n \times n$ symmetric matrix $A$ has a unique decomposition as a difference of two positive semidefinite matrices that are orthogonal to each other:

$$
A=Q-P, \text { with } Q, P \in \mathcal{P}_{n} \text { and } Q P=0 .
$$

In fact, $Q$ is the projection of $A$ on $\mathcal{P}_{n}$ and $P$ is the projection of $-A$ on the same cone.

More explicitly, let $\Lambda$ be the multiset of eigenvalues of $A$, and for every $\lambda \in \Lambda$ denote by $E_{\lambda}$ the orthogonal projection on the eigenspace of $\lambda$. Then

$$
A=\sum_{\lambda \in \Lambda} \lambda E_{\lambda}
$$

is the spectral decomposition of $A$.

Denote by $\Lambda_{+}$and $\Lambda_{-}$the multisets of positive and negative eigenvalues of $A$, respectively. Then $Q=\sum_{\lambda \in \Lambda_{+}} \lambda E_{\lambda}$ and $P=-\sum_{\lambda \in \Lambda_{-}} \lambda E_{\lambda}$. In particular, the 
spectrum of $Q$ consists of the elements of $\Lambda_{+}$together with $n-\left|\Lambda_{+}\right|$zeros and the spectrum of $P$ consists of the absolute values of the elements in $\Lambda_{-}$together with $n-\left|\Lambda_{-}\right|$zeros. We refer to $Q$ and $P$ as the positive definite part and the negative definite part of $A$, respectively.

If $A$ is not positive semidefinite, then obviously $A \neq 0$ and $P \neq 0$, and the cosine of the angle between $A$ and $P$ is

$$
\frac{\langle A, P\rangle}{\|A\| \cdot\|P\|}=\frac{-\langle P, P\rangle}{\|A\| \cdot\|P\|}=-\frac{\|P\|}{\|A\|}=-\frac{\sqrt{\sum_{\lambda \in \Lambda_{-}} \lambda^{2}}}{\sqrt{\sum_{\lambda \in \Lambda} \lambda^{2}}} .
$$

For every nonzero symmetric $n \times n$ matrix $A$, let us denote by $\angle\left(A, \mathcal{P}_{n}\right)$ the maximal angle between $A$ and a matrix in $\mathcal{P}_{n}$. The following holds:

Proposition 2. For every $A \in \mathcal{S}_{n} \backslash \mathcal{P}_{n}$, let $P \in \mathcal{P}_{n}$ be the negative definite part of A. Then

$$
\angle\left(A, \mathcal{P}_{n}\right)=\angle(A, P)=\arccos \left(-\frac{\sqrt{\sum_{\lambda \in \Lambda_{-}} \lambda^{2}}}{\sqrt{\sum_{\lambda \in \Lambda} \lambda^{2}}}\right),
$$

where $\Lambda$ and $\Lambda_{-}$are as described above. Moreover, $P$ is the unique matrix in $\mathcal{P}_{n}$, up to multiplication by a positive scalar, which forms this maximal angle with A.

Proof. For every $0 \neq X \in \mathcal{P}_{n}$ we have

$$
\frac{\langle A, X\rangle}{\|A\| \cdot\|X\|} \geq-\frac{\langle P, X\rangle}{\|A\| \cdot\|X\|} \geq-\frac{\|P\|}{\|A\|}=\frac{\langle A, P\rangle}{\|A\| \cdot\|P\|},
$$

where the first inequality follows from the fact that $Q$, the positive definite part of $A$, satisfies $\langle Q, X\rangle \geq 0$, and the second inequality from the Cauchy-Schwarz inequality. This shows that $\angle(A, X) \leq \angle(A, P)$ for every $X \in \mathcal{P}_{n}$. By the condition for equality in the Cauchy-Schwarz inequality, we get that $\angle(A, X)=\angle(A, P)$ if and only if $X$ is a positive scalar multiple of $P$.

Similarly, every $A \in \mathcal{S}_{n}$ has a unique decomposition as a difference of two nonnegative matrices that are orthogonal to each other:

$$
A=M-N, \text { with } M, N \in \mathcal{N}_{n} \text { and } M \circ N=0,
$$

where $\circ$ denotes the entrywise product of matrices (also often called the Hadamard product).

In fact, $M=\max (A, 0)$, with the maximum defined entrywise, is the projection of $A$ on $\mathcal{N}_{n}$, and $N=\max (-A, 0)$ is the projection of $-A$ on that cone. We refer to 
$M$ and $N$ as the positive part and the negative part of $A$, respectively. If $A \notin \mathcal{N}_{n}$, then $A, N \neq 0$, and the cosine of the angle between $A$ and $N$ is

$$
\frac{\langle A, N\rangle}{\|A\| \cdot\|N\|}=\frac{-\langle N, N\rangle}{\|A\| \cdot\|N\|}=-\frac{\|N\|}{\|A\|}=-\frac{\sqrt{\sum_{a_{i j}<0} a_{i j}^{2}}}{\sqrt{\sum a_{i j}^{2}}} .
$$

We denote by $\angle\left(A, \mathcal{N}_{n}\right)$ the maximal angle between $A$ and a matrix in $\mathcal{N}_{n}$. Then the following holds:

Proposition 3. For every $A \in \mathcal{S}_{n} \backslash \mathcal{N}_{n}$, let $N \in \mathcal{P}_{n}$ be the negative part of $A$. Then

$$
\angle\left(A, \mathcal{N}_{n}\right)=\angle(A, N)=\arccos \left(-\frac{\sqrt{\sum_{a_{i j}<0} a_{i j}^{2}}}{\sqrt{\sum a_{i j}^{2}}}\right) .
$$

Moreover, $N$ is the unique matrix in $\mathcal{N}_{n}$, up to multiplication by a positive scalar, which forms this maximal angle with $A$.

The proof is completely parallel to the proof of Proposition 2.2, and is therefore omitted. The next proposition demonstrates the computation of $\angle\left(P, \mathcal{N}_{n}\right)$ in a special case.

Proposition 4. Let $P \in \mathcal{P}_{n} \backslash \mathcal{N}_{n}$ have rank 1. Then $\angle\left(P, \mathcal{N}_{n}\right) \leq \frac{3}{4} \pi$. Furthermore, there exists a rank 1 positive semidefinite matrix $P \in \mathcal{P}_{n} \backslash \mathcal{N}_{n}$ such that $\angle\left(P, \mathcal{N}_{n}\right)=$ $\frac{3}{4} \pi$.

Proof. By the assumptions, $P=u u^{T}$, where $u$ has both positive and negative entries. By a suitable permutation of rows and columns of $P$ we may assume that

$$
u=\left[\begin{array}{r}
v \\
-w
\end{array}\right], \quad v, w \geq 0, \quad v, w \neq 0 .
$$

Then

$$
P=\left[\begin{array}{rr}
v v^{T} & -v w^{T} \\
-w v^{T} & w w^{T}
\end{array}\right],
$$

and the negative part of $P$ is

$$
N=\left[\begin{array}{cc}
0 & v w^{T} \\
w v^{T} & 0
\end{array}\right] .
$$

For any two vectors $x$ and $y$,

$$
\left\|x y^{T}\right\|=\sqrt{\operatorname{Tr}\left(x y^{T} y x^{T}\right)}=\|x\|\|y\| .
$$


Thus

$$
\|P\|=\|u\|^{2}=\|v\|^{2}+\|w\|^{2}, \quad\|N\|=\sqrt{2}\|v\| \cdot\|w\|,
$$

and

$$
\langle P, N\rangle=-2\left\|v w^{T}\right\|^{2}=-2\|v\|^{2}\|w\|^{2} .
$$

Thus

$$
\frac{\langle P, N\rangle}{\|P\| \cdot\|N\|}=-\frac{\sqrt{2}|| v\|\cdot\| w \|}{\|v\|^{2}+\|w\|^{2}} \geq-\frac{\sqrt{2}}{2}
$$

Equality holds in the last inequality if and only if $\|v\|=\|w\|$. Thus $\angle(P, N) \leq \frac{3}{4} \pi$, with equality if and only if $\|v\|=\|w\|$.

In particular, the last proposition implies the following known result (known by the proof of Proposition 6.15 in [12], and the monotonicity of $\left.\left\{\gamma_{n}\right\}\right)$.

Corollary 5. For every $n \geq 2, \gamma_{n} \geq \frac{3}{4} \pi$.

We can now prove

Proposition 6. Let $n \geq 2$, and let $P \in \mathcal{P}_{n}$ and $N \in \mathcal{N}_{n}$ be any two matrices such that $\angle(P, N)=\gamma_{n}$. Then $\langle P, N\rangle<0, \operatorname{diag} N=0$, and $1 \leq \operatorname{rank} P \leq n-1$.

Proof. By Corollary $5, \gamma_{n} \geq \frac{3}{4} \pi$, and thus $\langle P, N\rangle<0$. This implies that $P \notin \mathcal{N}_{n}$ and $N \notin \mathcal{P}_{n}$. Since $\angle(P, N)$ is the maximal possible angle between a positive semidefinite and a nonnegative matrix of the same order, $N$ has to be the nonnegative matrix forming the maximal possible angle with $P$, and $P$ has to be the nonnegative matrix forming the maximal possible angle with $N$.

By the uniqueness parts in Propositions 2 and 3, $N$ is a positive scalar multiple of the negative part of $P$, and $P$ is a positive scalar multiple of the negative definite part of $N$. Since $\operatorname{diag} P \geq 0$ and $N$ is the negative part of $P$, we get that $\operatorname{diag} N=0$. By the Perron-Frobenius Theorem the nonzero $N$ has at least one positive eigenvalue, so its negative definite part $P$ satisfies $\operatorname{rank} P \leq n-1$.

Proposition 7. Let $n \geq 2$, let $N \in \mathcal{N}_{n}$ have $\operatorname{diag} N=0$ and let $P$ be its negative definite part. If $\operatorname{rank} P=n-1$, then $\angle\left(N, \mathcal{P}_{n}\right)<\frac{3}{4} \pi$.

Proof. By the assumptions on $N$, its eigenvalues are $\rho=\lambda_{1}>0$, and $n-1$ negative eigenvalues $\lambda_{2}, \ldots, \lambda_{n}$ with $\sum_{i=2}^{n} \lambda_{i}=-\rho$. By Proposition 2,

$$
\cos \angle\left(N, \mathcal{P}_{n}\right)=-\frac{\sqrt{\sum_{i=2}^{n} \lambda_{i}^{2}}}{\sqrt{\rho^{2}+\sum_{i=2}^{n} \lambda_{i}^{2}}} .
$$


The function $g\left(x_{2}, \ldots, x_{n}\right)=\sum_{i=2}^{n} x_{i}^{2}$ is convex, and thus attains its maximum on the compact convex set

$$
\Delta=\left\{\left(x_{2}, \ldots, x_{n}\right) \in \mathbb{R}^{n-1}: x_{i} \leq 0, i=2, \ldots, n-1, \text { and } \sum_{i=2}^{n} x_{i}=-\rho\right\}
$$

at an extreme point of this set, i.e., at a point $x$ such that $x_{i}=-\rho$ for some $i$ and $x_{j}=0$ for $j \neq i$. That is,

$$
\max _{x \in \Delta} g(x)=\rho^{2}
$$

The function $f(t)=-\sqrt{\frac{t}{\rho^{2}+t}}$ is decreasing on $[0, \infty)$, and thus $f\left(g\left(x_{2}, \ldots, x_{2}\right)\right)$ attains a minimum on $\Delta$ where $g$ attains its maximum, and $\min _{x \in \Delta} f(g(x))=-\sqrt{\frac{\rho^{2}}{2 \rho^{2}}}=$ $-\frac{\sqrt{2}}{2}$. Since $\cos \angle\left(N, \mathcal{P}_{n}\right)=f\left(g\left(\lambda_{2}, \ldots, \lambda_{n}\right)\right)$, and $\left(\lambda_{2}, \ldots, \lambda_{n}\right) \in \Delta$, we get that $\angle\left(N, \mathcal{P}_{n}\right) \leq \cos \left(\min _{x \in \Delta} f(g(x))\right)=\frac{3}{4} \pi$.

By the assumption that $\operatorname{rank} P=n-1$ we see that $\left(\lambda_{2}, \ldots, \lambda_{n}\right)$ is not an extreme point of $\Delta$, and since $g(x)$ is strictly convex on $\Delta$, it does not attain its maximum on $\left(\lambda_{2}, \ldots, \lambda_{n}\right)$, and neither does $\arccos (f(g(x)))$. Hence the strict inequality.

In other words, Proposition 7 tells us that if $(N, P)$ is a pair attaining $\gamma_{n}$, then we must have $\operatorname{rank} P \leq n-2$.

We can now show:

Theorem 8. For $n \leq 4, \gamma_{n}=\frac{3}{4} \pi$.

Proof. Propositions 4, 6 and 7 imply that $\gamma_{n}=\frac{3}{4} \pi$ for $n \leq 3$. It remains to consider the case of $n=4$. Also, by these propositions it suffices to consider $\angle\left(N, \mathcal{P}_{n}\right)$ for $N \in \mathcal{N}_{4}$ with $\operatorname{diag} N=0$ and a negative definite part $P$ of rank 2. Such $N$ has a Perron eigenvalue $\rho>0$, and its complete set of eigenvalues is

$$
\rho \geq \mu \geq 0>\lambda_{3} \geq \lambda_{4}
$$

where $\lambda_{3}+\lambda_{4}=-\rho-\mu$ and $\lambda_{4} \geq-\rho$. Then

$$
\cos \angle\left(N, \mathcal{P}_{n}\right)=-\frac{\sqrt{\lambda_{3}^{2}+\lambda_{4}^{2}}}{\sqrt{\rho^{2}+\mu^{2}+\lambda_{3}^{2}+\lambda_{4}^{2}}} .
$$

Similarly to the previous proof, we note that $g(x, y)=x^{2}+y^{2}$ is a convex function, and the set

$$
\Delta=\left\{(x, y) \in \mathbb{R}^{2}: 0 \geq x \geq y \geq-\rho \text { and } x+y=-\rho-\mu\right\}
$$


is a compact convex set. By the assumptions on $\rho$ and $\mu, \Delta$ is the line segment

$$
y=-\rho-\mu-x \quad, \quad-\frac{\rho+\mu}{2} \leq x \leq-\mu .
$$

Its extreme points are

$$
(-\mu,-\rho) \text { and }\left(-\frac{\rho+\mu}{2},-\frac{\rho+\mu}{2}\right)
$$

and the maximal of $g$ on $\Delta$ is the greater of

$$
g(-\mu,-\rho)=\mu^{2}+\rho^{2} \text { and } g\left(-\frac{\rho+\mu}{2},-\frac{\rho+\mu}{2}\right)=\frac{(\rho+\mu)^{2}}{2} .
$$

Thus

$$
\max _{(x, y) \in \Delta} g(x, y)=\mu^{2}+\rho^{2}
$$

and it is attained when $x=-\mu$ and $y=-\rho$. The function $f(t)=-\sqrt{\frac{t}{\rho^{2}+\mu^{2}+t}}$ is a decreasing function on $[0, \infty)$, and therefore $f(g(x, y))$ attains a minimum on $\Delta$ at $(-\mu,-\rho)$, and $\min _{(x, y) \in \Delta} f(g(x))=-\sqrt{\frac{\rho^{2}+\mu^{2}}{2\left(\rho^{2}+\mu^{2}\right)}}=-\frac{\sqrt{2}}{2}$. Since $\left(\lambda_{3}, \lambda_{4}\right) \in \Delta$, we get that $\angle\left(N, \mathcal{P}_{4}\right) \leq \arccos \left(\min _{(x, y) \in \Delta} f(g(x))\right)=\frac{3}{4} \pi$. Together with Corollary 5 this completes the proof.

Note that he matrix

$$
N=\left[\begin{array}{llll}
0 & 1 & 0 & 0 \\
1 & 0 & 0 & 0 \\
0 & 0 & 0 & 1 \\
0 & 0 & 1 & 0
\end{array}\right]
$$

has eigenvalues $1,1,-1,-1$, and thus by the above argument $\gamma_{4}=\frac{3}{4} \pi$ is attained also by a pair $(N, P)$, where $P$ is the positive semidefinite part of $N$ and $\operatorname{rank} P=2$.

For $n=5$ the result of Theorem 8 no longer holds:

Example 9. Let

$$
N=\left[\begin{array}{lllll}
0 & 1 & 0 & 0 & 1 \\
1 & 0 & 1 & 0 & 0 \\
0 & 1 & 0 & 1 & 0 \\
0 & 0 & 1 & 0 & 1 \\
1 & 0 & 0 & 1 & 0
\end{array}\right]
$$

be the adjacency matrix of the 5-cycle. Its eigenvalues are well known (they are easily computed by the formula for the eigenvalues of a circulant matrix): the simple 
eigenvalue 2 , the positive eigenvalue $2 \cos (2 \pi / 5)=\frac{-1+\sqrt{5}}{2}$ of multiplicity 2 , and the negative eigenvalue $-2 \cos (\pi / 5)=\frac{-1-\sqrt{5}}{2}$ of multiplicity 2 . Thus the negative definite part $P$ of $N$ satisfies:

$$
\cos \angle(P, N)=-\frac{\sqrt{8 \cos ^{2}(\pi / 5)}}{\sqrt{4+8 \cos ^{2}(2 \pi / 5)+8 \cos ^{2}(\pi / 5)}}=-\frac{1+1 / \sqrt{5}}{2}<-\frac{\sqrt{2}}{2},
$$

implying that

$$
\gamma_{5} \geq \arccos \left(-\frac{1+1 / \sqrt{5}}{2}\right) \approx 0.7575 \pi>\frac{3}{4} \pi
$$

The negative definite part of $N$ is a scalar multiple of

$$
P=\left[\begin{array}{ccccc}
1 & -\cos (\pi / 5) & \cos (2 \pi / 5) & \cos (2 \pi / 5) & -\cos (\pi / 5) \\
-\cos (\pi / 5) & 1 & -\cos (\pi / 5) & \cos (2 \pi / 5) & \cos (2 \pi / 5) \\
\cos (2 \pi / 5) & -\cos (\pi / 5) & 1 & -\cos (\pi / 5) & \cos (2 \pi / 5) \\
\cos (2 \pi / 5) & \cos (2 \pi / 5) & -\cos (\pi / 5) & 1 & -\cos (\pi / 5) \\
-\cos (\pi / 5) & \cos (2 \pi / 5) & \cos (2 \pi / 5) & -\cos (\pi / 5) & 1
\end{array}\right]
$$

Indeed, the kind of argument that we used to prove Theorem 8 is no longer sufficient for the determination of $\gamma_{n}$ for $n \geq 5$. Here we present some considerations which explain the new difficulties which arise in the case $n \geq 5$.

Our proofs for the case $n \leq 4$ involved optimization of a convex function of the non-positive eigenvalues of a matrix $0 \neq N \in \mathcal{N}_{n}$ with zero diagonal, over a convex set formed by such eigenvalue-tuples. Continuing this line of proof for $n \geq 5$ would require some information on the possible sets of eigenvalues of a nonnegative $n \times n$ matrix with a zero diagonal. It is known that the eigenvalues

$$
\lambda_{1} \geq \lambda_{2} \geq \ldots \geq \lambda_{n}
$$

of a matrix $0 \neq N \in \mathcal{N}_{n}$ with zero diagonal satisfy

$$
\lambda_{1}>0, \quad \lambda_{n} \geq-\lambda_{1} \quad \text { and } \quad \sum_{i=1}^{n} \lambda_{i}=0 .
$$

But for $n \geq 5$ not all sequences satisfying (2.6) and (2.7) are eigenvalues of some such $N$. The problem of determining necessary and sufficient conditions for a set of real numbers to be the eigenvalues of some $N \in \mathcal{N}_{n}$ with a zero diagonal is part of the Symmetric Inverse Eigenvalue Problem (SNIEP), which is difficult and generally open. For $n \leq 4$ the conditions (2.6) and (2.7) are also sufficient, by results of 9$]$ and [15. For $n=5$ it is shown in [17 that necessary and sufficient conditions for (2.6) to 
be eigenvalues of some $N \in \mathcal{N}_{n}$ are (2.7) together with

$$
\lambda_{2}+\lambda_{5} \geq 0 \text { and } \sum_{i=1}^{5} \lambda_{i}^{3} \geq 0 .
$$

For $n \geq 6$ the SNIEP is still open even for trace zero matrices.

The solution of the trace-zero SNIEP for $n=5$ demonstrates a second difficulty in applying our approach, even for $n=5$ : The last condition in (2.8) is not redundant, and the set of all non-increasing 5 -tuples that are eigenvalues of a nonnegative trace zero matrix is not convex, complicating the relevant optimization problem. It seems that a new approach is needed for the computation of $\gamma_{n}, n \geq 5$.

For our purpose, of proving that $\lim _{n \rightarrow \infty} \gamma_{n}=\infty$, we will show that a judicious choice of a nonnegative matrix $N$ will allow the pair $(N, P)$, where $P$ is the negative definite part of the nonnegative matrix $N$, to attain ever higher angles. This will be done by taking $N$ as the adjacency matrix of a strongly regular graph.

3. Strongly regular graphs. Recall first the definition of strongly regular graphs, due originally to Bose, and the famous formula for the eigenvalues of such a graph.

Definition $10([5])$. A strongly regular graph with parameters $(n, k, a, c)$ is a $k$-regular graph on $n$ vertices such that any two adjacent vertices have $a$ common neighbours and any two non-adjacent vertices have $c$ common neighbours.

For instance, observe that the pentagon $C_{5}$ is strongly regular with parameters $(5,2,0,1)$ and that the Petersen graph is strongly regular with parameters $(10,3,0,1)$.

Obviously, not every quadruple of numbers $(a, b, c, d)$ is the parameter vector of a strongly regular graph. A number of necessary conditions are known and may be found in [10, Chapter 10]. We will only mention the simplest one, by way of illustration:

$$
(n-k-1) c=k(k-a-1) .
$$

The proof is an easy exercise in double counting.

The crucial fact for us here is that the eigenvalues of the adjacency matrix of a strongly regular graphs and their multiplicities depend only on the parameters (as there may often be many non-isomorphic graphs sharing the same parameters):

Theorem 11 (10, Section 10.2]). Let $G$ be a connected strongly regular graph with parameters $(n, k, a, c)$ and let $\Delta=(a-c)^{2}+4(k-c)$. The eigenvalues of the adjacency matrix $A(G)$ are: 
- $k$, with multiplicity 1.

- $\theta=\frac{(a-c)+\sqrt{\Delta}}{2}$, with multiplicity $m_{\theta}=\frac{1}{2}\left((n-1)-\frac{2 k+(n-1)(a-c)}{\sqrt{\Delta}}\right)$.

- $\tau=\frac{(a-c)-\sqrt{\Delta}}{2}$, with multiplicity $m_{\tau}=\frac{1}{2}\left((n-1)+\frac{2 k+(n-1)(a-c)}{\sqrt{\Delta}}\right)$.

Note that $m_{\theta}$ and $m_{\tau}$ have to be integers, and this is another necessary condition the parameters $(n, k, a, c)$ have to satisfy.

Let us now take $N$ to be the adjacency matrix of a strongly regular graph, and let be $P$ the negative definite part of $N$. Equation (2.1) takes on the following form then:

$$
\frac{\langle N, P\rangle}{\|N\| \cdot\|P\|}=-\sqrt{\frac{m_{\tau} \tau^{2}}{n k}}
$$

To complete the proof of Theorem 1 we would now like to exhibit a family of strongly regular graphs $\left\{G_{n_{k}}\right\}$ for which the expressions of (3.2) tend to -1 as $n_{k} \rightarrow$ $\infty$.

\section{Generalized quadrangles.}

Definition 12. A generalized quadrangle is a finite incidence structure $(\Pi, L)$ with sets $\Pi$ of points and $L$ of lines, such that:

- Two lines meet in at most one point.

- If $u$ is a point not on line $m$, then there are a unique point $v$ on $m$ and $a$ unique line $\ell$ such that $u$ and $v$ are on $\ell$.

For basic facts about generalized quadrangles we refer to [1, Chapter 6]. The advanced theory is laid out in [16]. Our definition here followed [6, p. 129].

If the generalized quadrangle $Q$ has the further property that every line is on $s+1$ points and every point is on $t+1$ lines, then we say that $Q$ is of order $(s, t)$ and denote it by $G Q(s, t)$. By [1, Theorem 6.1.1] all generalized quadrangles are either of this form or isomorphic to a grid or to a dual of a grid.

It is not known what are all the pairs $(s, t)$ for which a generalized quadrangle $G(s, t)$ exists. But the so-called "classical" constructions of generalized quadrangles, originally due to Tits, yields specimens of $G Q(s, 1), G Q(s, s)$ and $G Q\left(s, s^{2}\right)$ whenever $s=q$ is a prime power. (cf. [1, p. 118] and [6, pp. 130-131] for descriptions of these constructions.)

We need to introduce one final concept. The collinearity graph $C_{Q}$ of a generalized quadrangle $Q=(\Pi, L)$ has $\Pi$ for its vertex set and $u, v \in \Pi$ are adjacent in $C_{Q}$ if and only if $u$ and $v$ lie on a line in $Q$. 
Theorem 13 ([6, Theorem 9.6.2]). Let $Q$ be a generalized quadrangle of order $(s, t)$ and let $C_{Q}$ be its collinearity graph. Then $C_{Q}$ is strongly regular with parameters $(n, k, a, c)=((s+1)(s t+1), s(t+1), s-1, t+1)$ and its spectrum is:

- $k=s(t+1)$ with multiplicity 1 .

- $\theta=s-1$ with multiplicity $m_{\theta}=s t(s+1)(t+1) /(s+t)$.

- $\tau=-(t+1)$ with multiplicity $m_{\tau}=s^{2}(s t+1) /(s+t)$.

\section{Piecing everything together.}

Proof of Theorem [1. Let $\left\{n_{k}\right\}$ be the sequence of prime powers. For each $q \in\left\{n_{k}\right\}$ there exists a classical generalized quadrangle $Q_{k}$ of the $G Q\left(q, q^{2}\right)$ type. Let $N_{k}$ be the adjacency matrix of $C_{Q_{k}}$ and let $P_{k}$ be the projection of $\left(-N_{k}\right)$ on $\mathcal{P}_{n}$. Then the angle between $N_{k}$ and $P_{k}$ can be calculated with the help of Theorem 13 and (3.2): its cosine is

$$
-\sqrt{\frac{m_{\tau} \tau^{2}}{n k}}=-\sqrt{\frac{s(t+1)}{(s+1)(s+t)}}=-\frac{\sqrt{q^{2}+1}}{q+1}
$$

and this leads to

$$
\angle\left(N_{k}, P_{k}\right)=\arccos \left(-\frac{\sqrt{q^{2}+1}}{q+1}\right) \underset{q \rightarrow \infty}{\longrightarrow} \arccos (-1)=\pi .
$$

Since

$$
\pi>\theta_{\max }\left(\mathcal{C}_{n_{k}}\right) \geq \gamma_{n_{k}} \geq \angle\left(N_{k}, P_{k}\right) \text { for every } k,
$$

this implies $\lim _{k \rightarrow \infty} \theta_{\max }\left(\mathcal{C}_{n_{k}}\right)=\lim _{k \rightarrow \infty} \gamma_{n_{k}}=\pi$, and by the monotonicity of the sequences $\left\{\theta_{\max }\left(\mathcal{C}_{n}\right)\right\}$ and $\left\{\gamma_{n}\right\}$ the result follows.

Note that we did not actually find the value of $\gamma_{n}$ for every $n$, which is why we refer to our result as the asymptotic solution of the Hiriart-Urruty and Seeger problem.

To get a feel for the sequence of angles $\left\{\angle\left(N_{k}, P_{k}\right)\right\}$, we list here the first five elements in the sequence. The first five prime powers (our q's) are 2, 3, 4,5 and 7. The first five orders of the matrix pairs we generate are: $n_{1}=27, n_{2}=112, n_{3}=325$, $n_{4}=756$ and $n_{5}=2752\left(n=(q+1)\left(q^{3}+1\right)\right)$. Table 1 shows the lower bounds on $\gamma_{n}$ (and thus on $\theta_{\max }\left(\mathcal{C}_{n}\right)$ ) for these orders, computed using (5.1).

\begin{tabular}{c|c|c|c|c}
$n=27$ & $n=112$ & $n=325$ & $n=756$ & $n=2752$ \\
\hline $\arccos \left(-\frac{\sqrt{5}}{3}\right)$ & $\arccos \left(-\frac{\sqrt{10}}{4}\right)$ & $\arccos \left(-\frac{\sqrt{17}}{5}\right)$ & $\arccos \left(-\frac{\sqrt{26}}{6}\right)$ & $\arccos \left(-\frac{\sqrt{50}}{8}\right)$ \\
$\approx 0.7677 \pi$ & $\approx 0.7902 \pi$ & $\approx 0.8086 \pi$ & $\approx 0.8232 \pi$ & $\approx 0.8451 \pi$
\end{tabular}

Table 1: Lower bounds on $\gamma_{n}$ and $\theta_{\max }\left(\mathcal{C}_{n}\right)$ 


\section{A few remarks.}

1. Theorem 1 implies that for large $n$ there exist a nonnegative matrix and a positive semidefinite matrix that are almost opposite, and the cones $\mathcal{P}_{n}+\mathcal{N}_{n}$ and $\mathcal{C}_{n}$ are "barely pointed".

2. We do not know whether the pair $\left(N_{k}, P_{k}\right)$ constructed is actually antipodal in either $\mathcal{C}_{n_{k}}$ or $\mathcal{P}_{n_{k}}+\mathcal{N}_{n_{k}}$. However, it is not hard to check that this pair satisfies the weaker property of being a critical pair in $\mathcal{P}_{n_{k}}+\mathcal{N}_{n_{k}}$, as defined in [12, Definition 6.11]. Any antipodal pair is critical but not all critical pairs are antipodal. It is not obvious that this pair is a critical pair for $\mathcal{C}_{n_{k}}$.

Question. Is $\theta_{\max }\left(\mathcal{C}_{n}\right)=\gamma_{n}$ ? In other words, is the maximal angle in $\mathcal{C}_{n}$ always achieved by a nonnegative matrix and a positive semidefinite matrix?

In fact, we do not even know the answer to the following, ostensibly simpler, question:

Question. Is $\theta_{\max }\left(\mathcal{P}_{n}+\mathcal{N}_{n}\right)=\gamma_{n}$ ?

This is true for $n=2$ by the results of [12].

3. Hiriart-Urruty and Seeger [12, Proposition 6.15] found that the (unique up to multiplication by a positive scalar) pair of antipodal matrices in $\mathcal{C}_{2}$ is:

$$
X=\frac{1}{2}\left(\begin{array}{cc}
1 & -1 \\
-1 & 1
\end{array}\right), Y=\frac{\sqrt{2}}{2}\left(\begin{array}{ll}
0 & 1 \\
1 & 0
\end{array}\right) .
$$

This example is in fact a special case of our construction: $Y$ can be thought of as the normalized adjacency matrix of the complete graph $K_{2}$ and $X$ is the negative definite part of $Y$. The right-hand side of (2.1) equals $-\frac{\sqrt{2}}{2}$ in this case, as can be easily verified.

We observe that pairs of matrices that yield $-\frac{\sqrt{2}}{2}$ in (2.1), and thus an angle of $\frac{3}{4} \pi$, can be easily constructed for every order by taking $N$ as the adjacency matrix of a bipartite graph, by the Coulson-Rushbrooke Theorem on the symmetry of their spectra (cf. [3, p. 11]).

Another kind of pair which attains the angle $\frac{3}{4} \pi$ can be constructed for a prime power $q$ by taking $n=(q+1)\left(q^{2}+1\right)$ and letting $N$ be the adjacency matrix of $C_{G Q(q, q)}$, which is clearly not a bipartite graph.

7. Acknowledgements. We would like to thank the referee for helpful comments and especially for urging us to investigate $\gamma_{n}$ beyond what has been done in the original version of the paper, thus leading us to the discovery of Theorem 8

\section{REFERENCES}

[1] Lynn Margaret Batten. Combinatorics of finite geometries. Cambridge University Press, 2nd edition, 1997. 
[2] Abraham Berman and Robert J. Plemmons. Nonnegative Matrices in the Mathematical Sciences, volume 9 of Classics in Applied Mathematics. SIAM, 1994.

[3] Norman Biggs. Algebraic Graph Theory. Cambridge Mathematical Library. Cambridge University Press, 2nd edition, 1993.

[4] Immanuel M. Bomze, Werner Schachinger, and Gabriele Uchida. Think co(mpletely )positive! matrix properties, examples and a clustered bibliography on copositive optimization. $J$. Glob. Optim., 52:423-445, 2012.

[5] R.C. Bose. Strongly regular graphs, partial geometries and partially balanced designs. Pacific J. Math., 13:389-419, 1963.

[6] Andries E. Brouwer and Willem H. Haemers. Spectra of Graphs, volume 223 of Universitext. Springer, 2012.

[7] P. H. Diananda. On non-negative forms in real variables some or all of which are non-negative. Proc. Camb. Philos. Soc., 58:17-25, 1962.

[8] Mirjam Dür. Copositive Programming a Survey Recent Advances in Optimization and its Applications in Engineering. In Moritz Diehl, Francois Glineur, Elias Jarlebring, and Wim Michiels, editors, Recent Advances in Optimization and its Applications in Engineering, chapter 1, pages 3-20. Springer Berlin Heidelberg, Berlin, Heidelberg, 2010.

[9] Miroslav Fiedler. Eigenvalues of nonnegative symmetric matrices. Linear Algebra Appl., 9:119$142,1974$.

[10] Chris Godsil and Gordon Royle. Algebraic Graph Theory, volume 207 of Graduate Texts in Mathematics. Springer, 2001.

[11] Roland Hildebrand. The extreme rays of the $5 \times 5$ copositive cone. Linear Algebra Appl., 437(7):1538-1547, 2012.

[12] J.-B. Hiriart-Urruty and Alberto Seeger. A variational approach to copositive matrices. SIAM Review, 52(4):593-629, 2010.

[13] Alfredo Iusem and Alberto Seeger. On pairs of vectors achieving the maximal angle of a convex cone. Math. Program., Ser. B, 104(2-3):501-523, 2005.

[14] Alfredo Iusem and Alberto Seeger. Searching for critical angles in a convex cone. Math. Program., Ser. B, 120(1):3-25, 2009.

[15] Raphael Loewy and David London. A note on an inverse problem for nonnegative matrices. Linear and Multilinear Algebra, 6:83-90, 1978.

[16] Stanley E. Payne and Joseph A. Thas. Finite Generalized Quadrangles. EMS Series of Lectures in Mathematics. European Mathematical Society, second edition, 2009.

[17] Oren Spector. A characterization of trace zero symmetric nonnegative $5 \times 5$ matrices. Linear Algebra Appl., 434:1000-1017, 2011. 